\title{
Synthesis of Ag and Cd nanoparticles by nanosecond pulsed-discharge in liquid nitrogen
}

Running title: Synthesis of $\mathrm{Ag}$ and $\mathrm{Cd}$ nanoparticles by pulsed-discharge in liquid nitrogen

M. Trad ${ }^{1}$, A. Nominé ${ }^{1}$, N. Tarasenka ${ }^{2}$, J. Ghanbaja $^{1}$, C. Noël ${ }^{1}$, M. Tabbal ${ }^{3}$, Belmonte $(\bowtie)^{1}$

1 Université de Lorraine, CNRS, IJL, F-54000 Nancy,France

2 B.I. Stepanov Institute of Physics, National Academy of Sciences of Belarus

3 Department of Physics, American University of Beirut, Riad El Solh 1107 2020, Beirut, Lebanon

Email: thierry.belmonte@univ-lorraine.fr

Tel.: + 33372742499

Abstract The synthesis of $\mathrm{CdO}, \mathrm{Ag}_{2} \mathrm{O}(5 \mathrm{~nm})$ and $\mathrm{Ag}(\sim 20-30 \mathrm{~nm})$ nano-objects is achieved simultaneously by nanosecond-pulsed discharges in liquid nitrogen between one cadmium electrode and one silver electrode. Oxidation occurs when liquid nitrogen is fully evaporated and nanoparticles are in contact with the air. No alloy is formed, whatever the conditions, even though both elements are present simultaneously, as showed by timeresolved optical emission spectroscopy. This lack of reactivity between elements is attributed to the high pressure within the discharge that keeps each metallic vapor around the electrode it comes from. Each element exhibits a specific behavior. Cubic Cd particles, formed at $4 \mathrm{kV}$, get elongated with filamentary tips when the applied voltage reaches 7 and $10 \mathrm{kV}$. Cd wires are formed by assembly in liquid nitrogen of $\mathrm{Cd}$ nanoparticles driven by dipole assembly, and not by dielectrophoresis. On the contrary, silver spherical particles get assembled into 2D dendritic structures. The anisotropic growth of these structures is assumed to be due to the existence of pressure gradients.

Keywords Spark discharges, Submerged discharges, Time-resolved optical emission spectroscopy, Liquid nitrogen

\section{$1 \quad$ Introduction}

Bimetallic nanoparticles (NPs), composed of two different metallic elements, are of greater interest than monometallic ones, from both scientific and technological views, to improve catalytic properties [1]. It was shown that even linked, monometallic nanoparticles exhibit improved catalytic properties with respect to separated one [2]. Creating nanosecond-pulsed 
discharges between two electrodes immerged in a dielectric liquid is a competitive process to produce nanoparticles at high rate. It is less expansive than laser ablation [3]. However, the way alloy nanoparticles are created in non-equilibrium discharges submitted to huge gradients in time and space is still unclear, especially in the case of electrodes made of different metals. Several attempts to synthesize alloy NPs by discharges in liquids were recently reported. For instance, Pootawang et al. [4] used DC pulsed discharges between Pt and Ag electrodes in a solution containing water, $\mathrm{NaCl}$ and sodium dodecylsulfonate. They obtained $\mathrm{Ag}-\mathrm{Pt}$ bimetallic particles in the form of nanocomposites and $\mathrm{Pt}$ oxide NPs. From $\mathrm{Co}$ and $\mathrm{Ag}$ electrodes immersed in a $0.1 \mathrm{M}(\mathrm{HCl}$ and $\mathrm{NaOH})$ electrolyte, Chang et al. [5] combined a DC pulsed excitation with ultrasonic vibration (at $\sim 20 \mathrm{kHz}$ ). They got a mixture of linked $\mathrm{Ag}$ and Co NPs with an average size of $30 \mathrm{~nm}$.

After Ag-Pt and Ag-Co, choice mas made to study the Ag-Cd system. Indeed, from seminal works in this field [6,7], erosion is proposed to be caused by electrode melting and evaporation, which produces the metallic vapor whose condensation leads to the synthesis of nanoparticles. In the $\mathrm{Ag}-\mathrm{Cd}$ system, the melting point of $\mathrm{Cd}$ is much lower than the melting point of Ag, contrary to the two other cases. This system is then interesting to better understand the way nanoparticles are formed by discharges in liquids.

Liquid nitrogen is an excellent dielectric. The main advantage of using this liquid in the experimental conditions that were used in this work is that no nitrogen lines are emitted. Only metallic lines are visible, which simplifies a lot interpretation of spectra. The choice of the cadmium-silver system originates from the difference in the melting temperatures of the two elements. Cadmium melts at $594.22 \mathrm{~K}$ whereas silver melts at $1234.93 \mathrm{~K}$. It is also interesting because many compounds are available in this system: For instance at $573 \mathrm{~K}$, one can find a silver-rich alloy with a maximum solubility of cadmium of $42 \%$. Beyond this content, the $\zeta$, $\gamma$ 'and $\varepsilon$ phases have compositions varying from 50 to 55 at. $\%$ of cadmium, 57 to 61 at. $\%$, and 66 to 81 at.\% of silver, respectively. A cadmium-rich alloy with a limited solubility of silver $(6.5 \%)$ is finally available close to pure cadmium. So, if any alloy forms by quenching from the liquid phase, one might identify easily the liquid phase composition.

After a short description of the experimental set-up, results obtained by time-resolved optical emission spectroscopy will be presented first to follow the way radiative transitions of both elements, cadmium and silver, evolve as a function of the pulse width. In a second time, material results will be presented. Then, we will sort among the possible mechanisms that drive the synthesis of nanoparticles in the present conditions and how they get assembled into anisotropic structures.

\section{Experimental setup}

The experimental set-up was presented in detail in a previous work of ours [8]. Briefly, a pinto-pin electrode configuration, immersed in liquid nitrogen, was used (Supplemental material 1). Electrodes were Cd (purity: 99.95\%) and Ag (purity: 99.99\%) wires (1 mm in diameter). A high DC voltage power supply (Technix SR15-R-1200-15 kV-80 mA) fed a solid-state switch (HTS-301-03-GSM) connected to one pin-electrode, the other electrode being grounded. The voltage rise time was $20 \mathrm{~ns}$ without any ballast resistor. In this work, the ballast resistance was null. The applied voltage of $+10 \mathrm{kV}$ and an inter-electrode gap distance of $100 \mu \mathrm{m}$ were chosen for all experiments. The inter-electrode distance is kept constant during the process by continuously adjusting it. This enables breakdown; otherwise the process stops. The pulse width was adjusted at 100, 500 and $2500 \mathrm{~ns}$ and period was set to 333 ms (Fig. 1). The voltage and current time evolutions were recorded simultaneously. The 
time $t=0$ is defined as the beginning of the current rise. The sharp voltage rise before breakdown (corresponding to an increase in the current intensity) followed by a plateau corresponds to charge injection in liquid nitrogen. As the voltage drops and oscillates weakly, the current rises and oscillates as well during the discharge step. The afterglow starts when the current is equal to zero, i.e. after the current drop and the few following oscillations. Then, if the pulse width is $100 \mathrm{~ns}$ (resp. 500 and $2500 \mathrm{~ns}$ ), the discharge time is $600 \mathrm{~ns}$ (resp. $900 \mathrm{~ns}$ and $2700 \mathrm{~ns}$ ) within an accuracy range of $\pm 50 \mathrm{~ns}$.

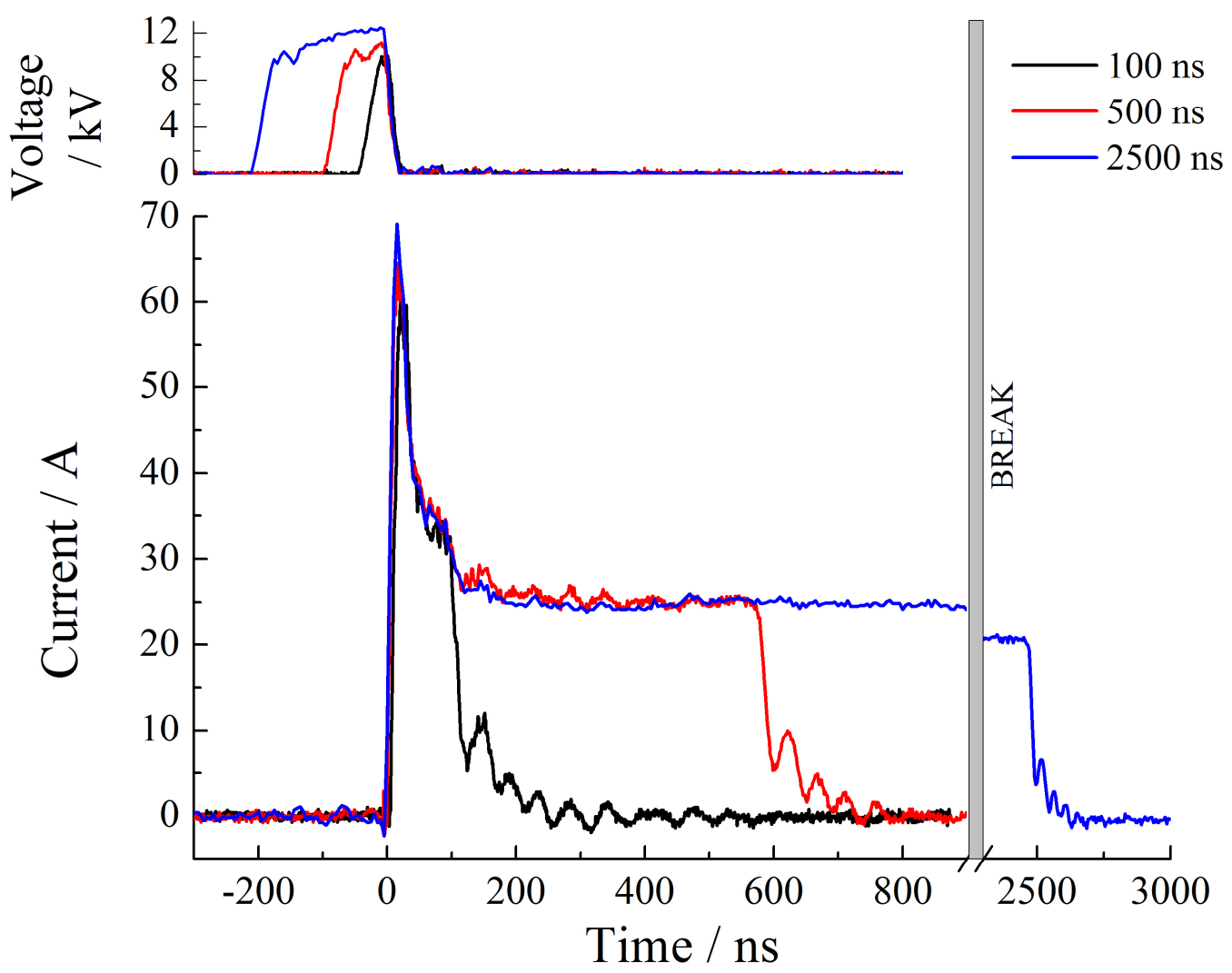

Fig. 1 Time-evolution of the voltage and current recorded for pulse width of 100, 500 and $2500 \mathrm{~ns}$.

Optical emission spectroscopy was performed with a $550 \mathrm{~mm}$ focal length monochromator (Jobin-Yvon TRIAX 550) equipped with a $100 \mathrm{~g} \mathrm{~mm}^{-1}$ grating for overall spectra in the (250-900 nm) visible range. It was coupled with a HORIBA Jobin-Yvoni-Spectrum Two iCCD detector. Each measurement is averaged over 50 spectra recorded with an exposure time of $50 \mathrm{~ns}$. Although discharges in dielectric liquids are known to be stochastic, the use of the solid-state switch ensures a high level of reproducibility because breakdown necessarily occurs within a time window inferior to the exposure time (see reference [9] for further details). However, because of the generation of high-frequency signals by discharge current oscillations (at about $1 \mathrm{MHz}$ ), ghost lines are sometimes observed. They are easily identified in time-resolved data, for they disappear from one spectrum to the other.

Nanoparticles are collected by sedimentation on a silicon wafer located under the pinelectrodes. We resorted to scanning electron microscopy (SEM) - XL30S-FEG by Philipsfor structural and chemical observations. The XL30S-FEG microscope was equipped with an EDXS (Energy-Dispersive X-ray Spectrometer) used for elemental analysis and a TLD detector (through the lens detector) used for high resolution imaging. A Philips CM200 device 
and a JEOL ARM 200F Cold FEG device were used for TEM investigation in order to study the crystallinity and the chemical composition of NPs deposited on copper grids.

\section{$3 \quad$ Optical emission spectroscopy}

Time-resolved optical emission spectra were recorded with an integration time of $50 \mathrm{~ns}$ for pulse widths equal to 100 and $2500 \mathrm{~ns}$ (Supplemental material2). Discharges were ignited between one electrode of cadmium and one electrode of silver for an applied voltage of +10 kV. In Fig. 2, a typical spectrum and the corresponding identification of line transitions are depicted (see Table 1 for a list). First, it is important to notice that emission lines are strongly broadened, shifted and sometimes distorted (their shape becomes asymmetric) especially at the beginning of the discharge because of the important contribution of the Stark effect, which is due to the high electron density in the discharge (typically around $10^{16} \mathrm{~cm}^{-3}$ ) [10]. If the presence of the Cd I and Cd II systems is easy to admit, no silver transition can be straightforwardly assessed from such spectra due to the overlapping (lines being broad and shifted) or to the proximity of $\mathrm{Ag}$ and $\mathrm{Ag}^{+}$lines that are known to be intense with $\mathrm{Cd}$ or $\mathrm{Cd}^{+}$ lines. The presence of the Ag I system in these spectra can only be established thanks to their corresponding time evolution. Indeed, when the discharge stops, ionic lines collapse and other emission lines get narrower. When looking at transitions around $520 \mathrm{~nm}$ in the spectrum given as Supplemental material3, we observe the narrowing of the $\mathrm{Cd}$ I line at $508.58 \mathrm{~nm}$ and the appearance of the Ag I line at $520.91 \mathrm{~nm}$ after about $1300 \mathrm{~ns}$, which becomes even clearer in the afterglow (i.e. beyond $2500 \mathrm{~ns}$ ). This does not mean that silver is not present at the very beginning of the discharge but simply that it cannot be detected in this case. In Supplemental material4, the time evolutions of Ag I lines at 328.07 and $338.29 \mathrm{~nm}$, which are by far more intense than any other silver line, are given for pulse widths of $100 \mathrm{~ns}$ and $2500 \mathrm{~ns}$. The lines are convoluted with other cadmium lines $(325.25,326.11 \mathrm{~nm}$ for the first one and $340.37 \mathrm{~nm}$ for the second one). A simplified method was used to estimate the area of the two selected silver lines (Supplemental material5). Finally, with time-resolved spectra, the presence of Ag II transitions could be assessed. 


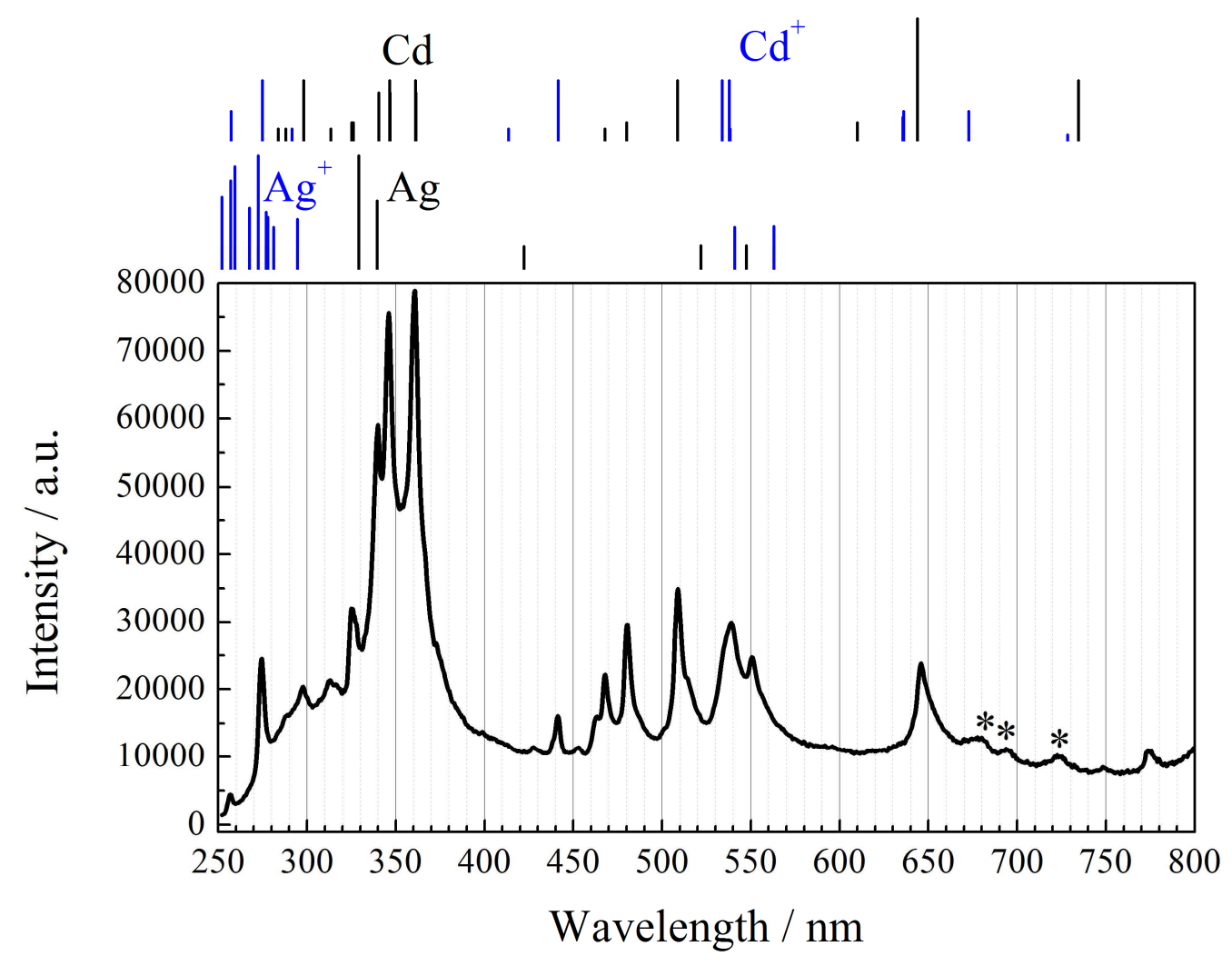

Fig. 2 Identification of emission lines of an emission spectrum recorded in the visible range $800 \mathrm{~ns}$ after breakdown. Theoretical transitions are materialized by ticks whose heights are proportional to their relative intensities normalized to the maximum value in the visible range. Acquisition time: $50 \mathrm{~ns}$. Applied voltage: $+10 \mathrm{kV}$. Pulse width: $2500 \mathrm{~ns}$. If Cd I and Cd II transitions are clearly present, the presence of Ag I transitions cannot be asserted with this spectrum and it requires results presented in Supplemental Material 3. Stars denote secondorder transitions.

In Figs. 3a and 3b, the time evolution of selected lines is depicted: three from $\mathrm{Cd}^{+}$, two from $\mathrm{Cd}$ and two from Ag. At short pulse width (Fig. 3a), ionic lines are observed as long as the current is not null. Their evolution roughly follows the shape of the current. Neutral lines decay exponentially and are visible in the afterglow that lasts about 150-200 ns. All selected neutral transitions evolve with very similar time constants. For a so short pulse width, the emission line of silver at $546.55 \mathrm{~nm}$ could only be detected during the afterglow because the presence of this line earlier in the spectrum could not be confirmed. In Fig. 3b, the time evolution of the selected emission lines is depicted for a pulse width of 2500 ns. Ionic lines decay slowly, in accordance with the description made previously: they follow the behavior of the current and they fade away in the afterglow period. Neutral lines are almost constant during the current plateau and decay exponentially in the afterglow, as already observed for a pulse width of $100 \mathrm{~ns}$. Here again, time constants are almost the same. The progressive appearance of silver transitions at $328.07 \mathrm{~nm}$ and $546.55 \mathrm{~nm}$ is materialized by a dotted line. The presence of silver atoms during the discharge is then established and it is possible to know that emission of silver starts as early as cadmium (within a precision range of $50 \mathrm{~ns}$ ). 
Table 1: Identified transitions corresponding to emission lines depicted in Fig. 2 (unresolved double peaks are denoted with brackets). For lines written in italic, see Supplemental Material 3 for their identifications. Second-order lines are written $2 \times \lambda$.

\begin{tabular}{|c|c|c|c|}
\hline Transition & Wavelength (nm) & Upper level & Lower level \\
\hline $\mathrm{Cd}$ II & 257.29 & $6 \mathrm{~s}^{2} \mathrm{~S}_{1 / 2}$ & $5 p^{2} \mathrm{P}^{\circ}{ }_{1 / 2}$ \\
\hline Cd II & 274.85 & $6{ }^{2} S_{1 / 2}$ & $5 \mathrm{p}^{2} \mathrm{P}^{\circ} 3 / 2$ \\
\hline $\mathrm{Cd} \mathrm{I}$ & $298.06(298.14)$ & $5 s 6 s^{3} \mathrm{D} J=3$ & $5 s 5 p^{3} \mathrm{P}^{\circ} \mathrm{J}=2$ \\
\hline $\mathrm{Cd} \mathrm{I}$ & 313.32 & $5 s 7 s{ }^{3} \mathrm{~S} \mathrm{~J}=1$ & $5 \mathrm{~s} 5 \mathrm{p}^{3} \mathrm{P}^{\circ} \mathrm{J}=1$ \\
\hline $\mathrm{Cd} \mathrm{I}$ & 325.25 & $5 \mathrm{~s} 7 \mathrm{~s}{ }^{3} \mathrm{~S} J=1$ & $5 s 5 p^{3} \mathrm{P}^{\circ} \mathrm{J}=2$ \\
\hline $\mathrm{Cd} \mathrm{I}$ & 326.11 & $5 s^{21} S J=0$ & $5 \mathrm{~s} 5 \mathrm{p}^{3} \mathrm{P}^{\circ} \mathrm{J}=1$ \\
\hline$A g I$ & 328.07 & $5 p^{2} P_{3 / 2}^{\circ}$ & $5 s^{2} S_{1 / 2}$ \\
\hline AgI & 338.29 & $5 p^{2} P_{1 / 2}^{\circ}$ & $5 s^{2} S_{1 / 2}$ \\
\hline $\mathrm{Cd} \mathrm{I}$ & 340.37 & $5 \mathrm{~s} 5 \mathrm{~d}^{3} \mathrm{D} \mathrm{J}=1$ & $5 s 5 p^{3} \mathrm{P}^{\circ} \mathrm{J}=0$ \\
\hline $\mathrm{Cd} \mathrm{I}$ & $346.62(346.76)$ & $5 \mathrm{~s} 5 \mathrm{~d}^{3} \mathrm{D} \mathrm{J}=2$ & $5 s 5 p^{3} \mathrm{P}^{\circ} \mathrm{J}=1$ \\
\hline $\mathrm{Cd} \mathrm{I}$ & 361.05 (361.28) & $5 \mathrm{~s} 5 \mathrm{~d}^{3} \mathrm{D} \mathrm{J}=3$ & $5 \mathrm{~s} 5 \mathrm{p}^{3} \mathrm{P}^{\circ} \mathrm{J}=2$ \\
\hline Cd II & 441.56 & $5 s^{22} D_{5 / 2}$ & $5 \mathrm{p}^{2} \mathrm{P}_{3 / 2}^{\circ}$ \\
\hline $\mathrm{Cd} \mathrm{I}$ & 467.81 & $5 s 6 s{ }^{3} \mathrm{~S} \mathrm{~J}=1$ & $5 \mathrm{~s} 5 \mathrm{p}^{3} \mathrm{P}^{\circ} \mathrm{J}=0$ \\
\hline Cd II & $2 \times 226.50$ & $5 p^{2} \mathrm{P}_{1 / 2}^{\circ}$ & $5 s{ }^{2} S_{1 / 2}$ \\
\hline Cd II & $2 \times 231.28$ & $5 d^{2} D_{5 / 2}$ & $5 \mathrm{p}^{2} \mathrm{P}_{3 / 2}^{\circ}$ \\
\hline Cd II & $2 \times 274.85$ & $6{ }^{2} \mathrm{~S}_{1 / 2}$ & $5 \mathrm{p}^{2} \mathrm{P}_{3 / 2}^{\circ}$ \\
\hline $\mathrm{Cd} \mathrm{I}$ & 479.99 & $5 s 6 s{ }^{3} \mathrm{~S} \mathrm{~J}=1$ & $5 s 5 p^{3} \mathrm{P}^{\circ} \mathrm{J}=1$ \\
\hline$A g I$ & 487.41 & $5 s 6 s^{4} D_{7 / 2}$ & $5 s 5 p^{4} \mathrm{~F}_{9 / 2}^{\circ}$ \\
\hline Cd I & 508.58 & $5 s 6 s{ }^{3} \mathrm{~S} \mathrm{~J}=1$ & $5 s 5 p^{3} \mathrm{P}^{\circ} \mathrm{J}=2$ \\
\hline$A g I$ & 520.91 & $5 d^{2} D_{3 / 2}$ & $5 \mathrm{p}^{2} \mathrm{P}^{\circ} 1 / 2$ \\
\hline Cd II & 533.75 & $4 \mathrm{f}^{2} \mathrm{~F}^{\circ}{ }_{5 / 2}$ & $5 d^{2} D_{3 / 2}$ \\
\hline Cd II & $537.81(538.19)$ & $4 f^{2} \mathrm{~F}^{\circ} 7 / 2$ & $5 d^{2} D_{5 / 2}$ \\
\hline$A g I$ & 546.55 & $5 d^{2} D_{5 / 2}$ & $5 p^{2} P^{\circ}{ }_{3 / 2}$ \\
\hline $\mathrm{Cd} \mathrm{I}$ & 643.84 & $5 s 5 d^{1} D J=2$ & $5 \mathrm{~s} 5 \mathrm{p}^{1} \mathrm{P}^{\circ}$ \\
\hline $\mathrm{Cd} \mathrm{I}$ & $2 \times 340.37$ & $5 s 5 d^{3} D J=1$ & $5 s 5 p^{3} \mathrm{P}^{\circ} \mathrm{J}=0$ \\
\hline $\mathrm{Cd} \mathrm{I}$ & $2 \times 346.62(346.76)$ & $5 s 5 d^{3} D J=2$ & $5 \mathrm{~s} 5 \mathrm{p}^{3} \mathrm{P}^{\circ} \mathrm{J}=1$ \\
\hline $\mathrm{Cd} \mathrm{I}$ & $2 \times 361.05(361.28)$ & $5 s 5 d^{3} D J=3$ & $5 s 5 p^{3} \mathrm{P}^{\circ} \mathrm{J}=2$ \\
\hline
\end{tabular}



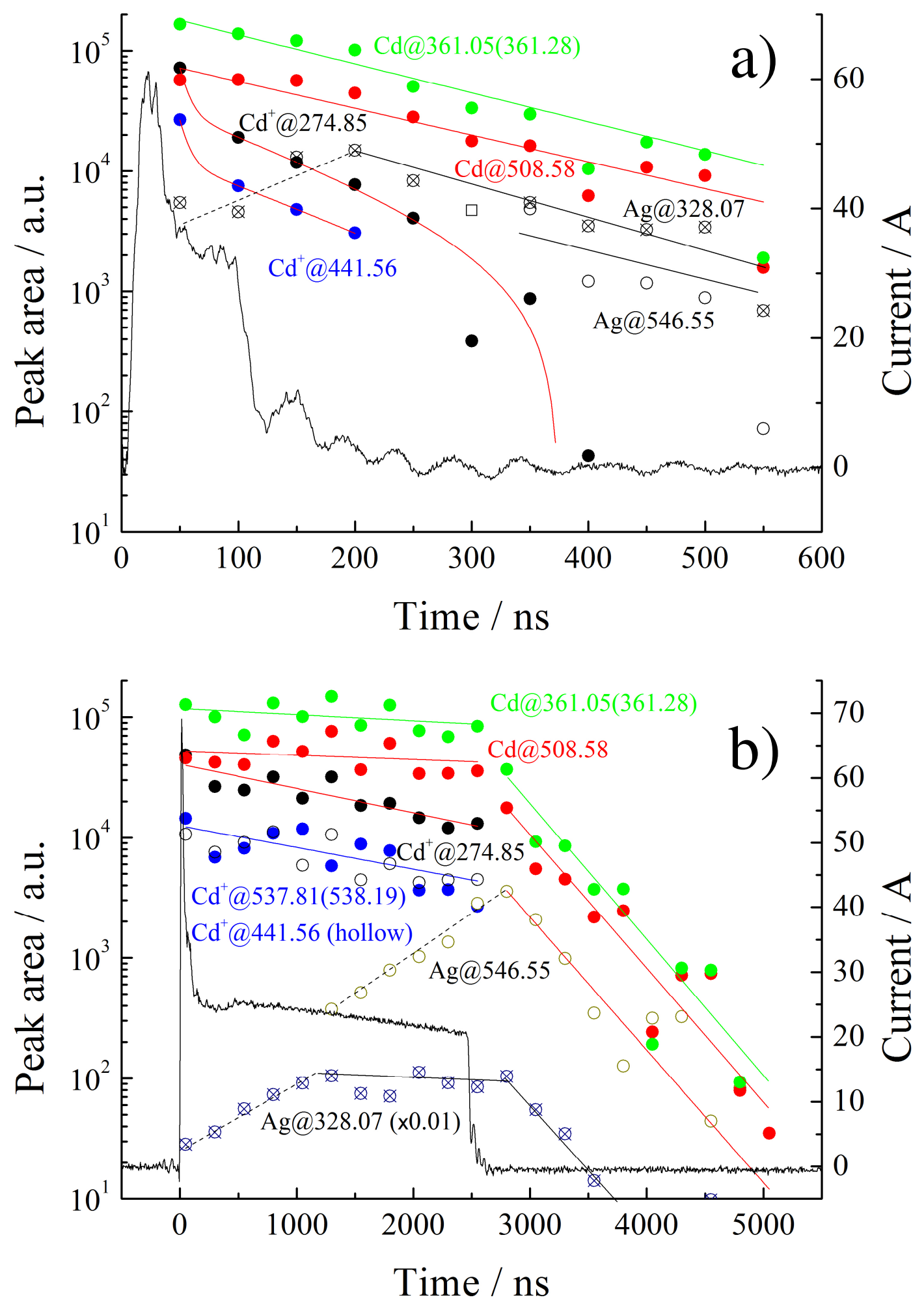

Fig. 3: Time evolution of selected emission lines a) for a pulse width of $100 \mathrm{~ns}$ (data recorded every $50 \mathrm{~ns}$ ) and b) for a pulse width of $2500 \mathrm{~ns}$ (data recorded every $250 \mathrm{~ns}$ ). Each area is the integrated value of the line intensity over a relevant window of wavelength. The dotted line represent the evolution of the area of the silver transition in the discharge period where this contribution starts emerging as a shoulder of its neighboring Cd I line. The current flowing through the plasma is also given (right scale). The evolution of the double transition at 537.81 $\mathrm{nm}$ and $538.19 \mathrm{~nm}$ was too weak to be evaluated and plotted in a). 
Treatments were performed by running discharges for one hour between one $\mathrm{Cd}$ electrode and one Ag electrode. The duration of the treatment defines the amount of nano-objects that are collected. Four pulse widths (100, 500, 1500 and $2500 \mathrm{~ns})$ and three applied voltages $(4,7$ and $10 \mathrm{kV}$ ) were used. At $10 \mathrm{kV}$ and beyond $100 \mathrm{~ns}$, the presence of three main kinds of nanoobjects is noticed: wires and elongated particles made of cadmium oxide on the one hand, and clusters made of silver on the other hand (Fig. 4). All these objects are assembly of nanoparticles with sizes around $5-10 \mathrm{~nm}$ for Cd-containing objects and around 30-50 nm for silver clusters, as shown in high-resolution images (Fig. 5 for wires and Supplemental materials 6 and 7 for $\mathrm{Cd}$ particles and silver clusters respectively). Silver nanoparticles are round-shaped whereas those made of cadmium are cubic (Fig. 5c) with sharp edges.

Micro-diffraction and micro-EDS analyses (see Supplemental materials 8 and 9) show that nano-objects are $\mathrm{CdO}$ for cadmium and $\mathrm{Ag}$ or $\mathrm{Ag}_{2} \mathrm{O}$ for silver. $\mathrm{AgO}$ is obtained if the time during which $\mathrm{Ag}$ nanoparticles are left in the air exceeds typically one day. In this latter case, only nanoparticles with sizes around $5 \mathrm{~nm}$ are found to be oxidized (see supplemental materials 8). Clusters, made of larger nanoparticles, are essentially metallic. Oxidation occurs once liquid nitrogen is evaporated, leaving nano-objects in contact with the air. To sum up, the composition of each object is reported in Table 2.

The pulse width affects the amount of nano-objects produced per pulse but it turns out that it does not significantly affect the shape of objects beyond 500 nanoseconds (Fig. 4).

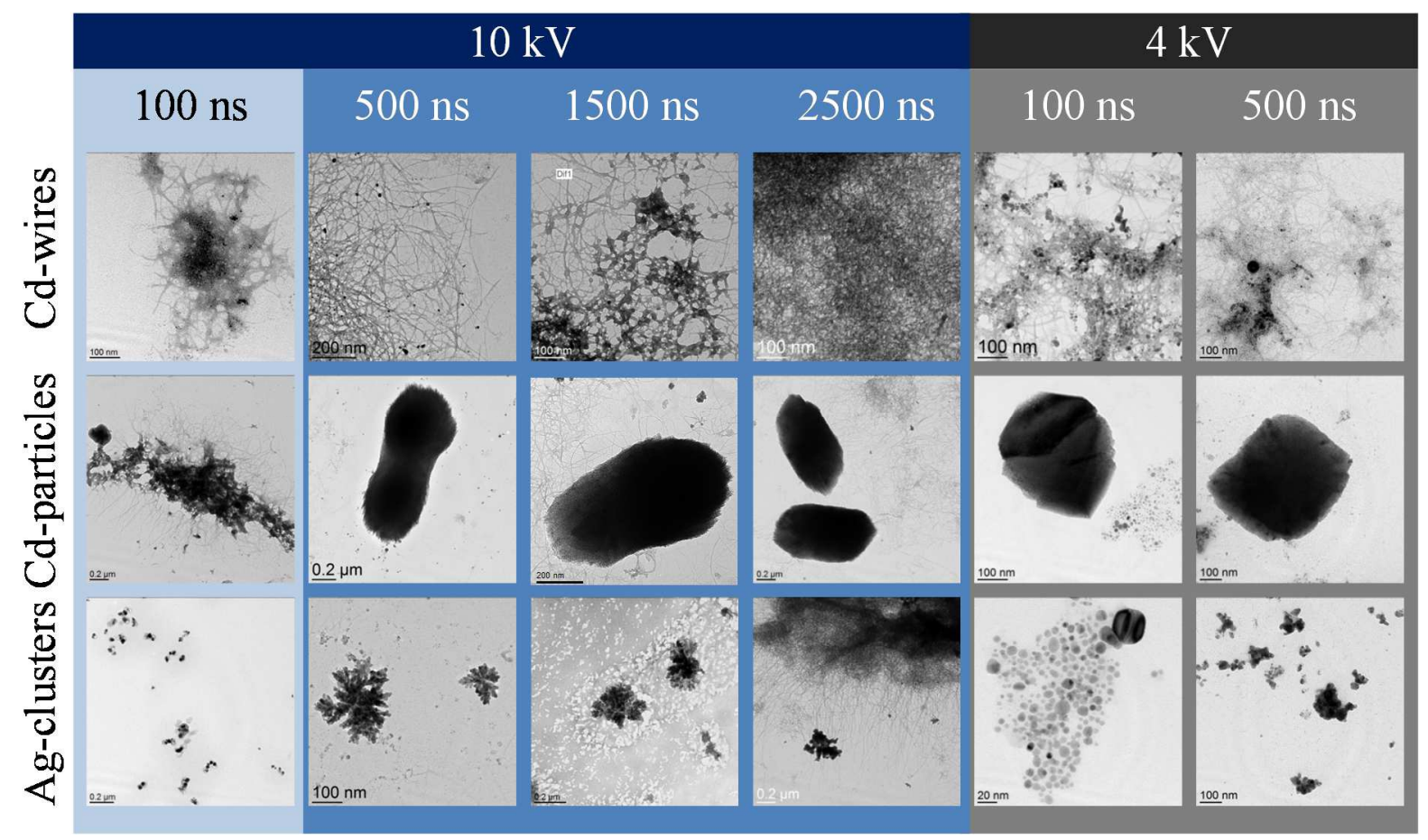

Fig. 4:Nano-objects synthesized at $10 \mathrm{kV}$ and $4 \mathrm{kV}$ for different pulse widths.

CdO nano-objects are visibly "stretched" when the applied voltage increases. This means that their initial cubic shape - we checked that these objects were 3D and not 2D- evolves towards the shape of an elongated object with both ends getting filamentary (Supplemental Material 10). These objects seem to be formed a bit later when the applied voltage is lower, contrary to silver clusters. 
Table 2: Composition of nano-objects in atomic percent.

\begin{tabular}{cccc}
\hline Nano-object & $\mathrm{Ag}$ & $\mathrm{Cd}$ & $\mathrm{O}$ \\
\hline CdO-Wires & Traces & $50 \pm 5$ at. $\%$ & $50 \pm 5$ at. $\%$ \\
CdO-elongated particles & Traces & $50 \pm 5$ at. $\%$ & $50 \pm 5$ at. $\%$ \\
$\mathrm{Ag}_{2} \mathrm{O}$-particles $<5 \mathrm{~nm}$ & $65 \pm 5$ at. $\%$ & Traces & $35 \pm 5$ at. $\%$ \\
Ag-clusters & $95 \pm 5$ at. $\%$ & Traces & Traces \\
\hline
\end{tabular}
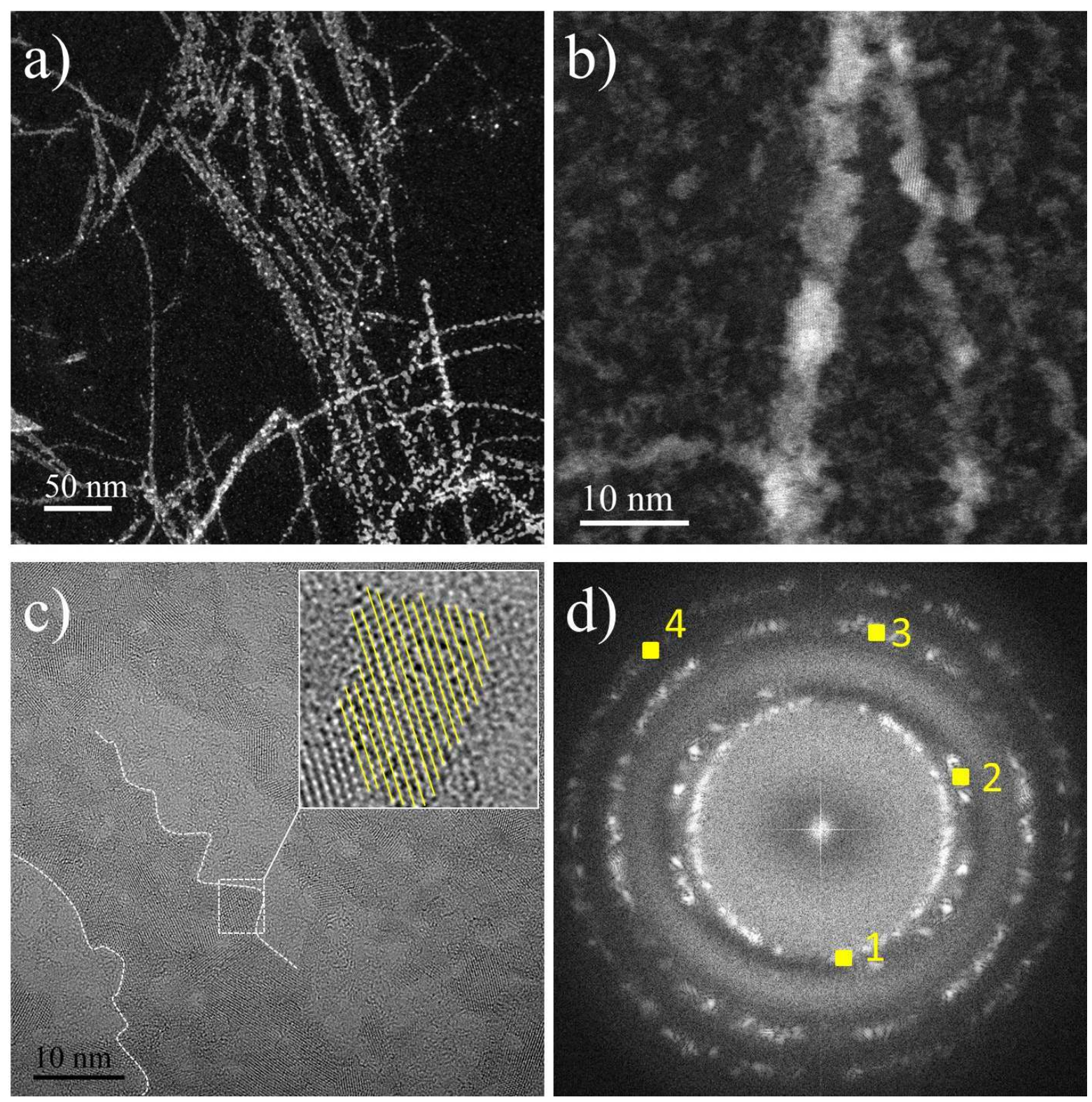

Fig. 5:a) large view STEM HAADF micrograph of a set of nanowires. b) Magnification showing individual nanowires. c) HRTEM micrograph of a single nanowire whose edges are showed by dotted lines. Inset: magnification of a nanoparticle composing the nanowire. Atomic planes are materialized by yellow lines. d) Fast Fourier transform image of the set of nanoparticles composing a nanowire. dot1: (111). dot2: (200). $\operatorname{dot} 3$ : (220). $\operatorname{dot} 4$ : (222) of cubic $\mathrm{CdO}(F m \overline{3} m)$. 


\subsection{Existing theories on metallic nanoparticle assembly}

There are not so many possible mechanisms to produce linear assembly of metallic nanoparticles. One is based on dielectrophoresis and one is based on dipole alignment.

In 2001, Hermanson et al. [11] showed the possibility to grow gold nanowires in an ac electric field from a suspension of gold nanoparticles by dielectrophoresis (also denoted DEP hereinafter). The dielectrophoretic force arises from the interaction of the particle dipoles with the non-uniform ac field and is directed along the field gradient:

$$
\vec{F}_{d e p}=\pi a^{3} \widetilde{\varepsilon}_{m} \Re \cdot \vec{\nabla}\left|\vec{E}_{r m s}\right|^{2}
$$

with $\omega$ the angular field frequency and $a$ the particle radius. $\vec{\nabla}\left|\vec{E}_{r m s}\right|^{2}$ is the gradient of the square of the RMS electrical field. $\Re$ denotes the real part of the Clausius-Mossotti factor, which is an effective polarizability of the particle in the medium. The absolute value of the force on a particle depends on $\vec{\nabla}\left|\vec{E}_{r m s}\right|^{2}$ and also on $\mathfrak{R}$. This latter parameter is expressed by the complex relative permittivities of the particle $\widetilde{\varepsilon}_{p}$ and the medium $\widetilde{\varepsilon}_{m}$ :

$$
\Re=\Re e\left(\frac{\widetilde{\varepsilon}_{p}(\omega)-\widetilde{\varepsilon}_{m}}{\widetilde{\varepsilon}_{p}(\omega)+2 \widetilde{\varepsilon}_{m}}\right)
$$

and it is bounded by the limits $1<\Re<-1 / 2$. It varies with the frequency of the applied field and the complex permittivity of the medium. Positive dielectrophoresis occurs when $\mathfrak{R}>0$, the force is towards points of high electrical field and the particles collect at the electrode edges. The converse of this is negative DEP which occurs when $\mathfrak{R}<0$, the force is in the direction of decreasing field strength and the particles are repelled from the electrode edges.

The ac field must be in the $\mathrm{MHz}$ range typically to trap nanoparticles with diameters between $10 \mathrm{~nm}$ and $100 \mathrm{~nm}$ [12].

Aggregation occurs only when the concentration of particles accumulated near the end of the wire is sufficiently high. Indeed, the dielectrophoretic force must overcome the electrostatic repulsion between particles to enable their aggregation. The dielectrophoretic particle transport to the tips of the wires is then of primary importance to sustain the wire growth and prevent depletion effects at the tips.

The frequency dependence of $\mathfrak{R}$ is given by:

$$
\Re=\left(\frac{\varepsilon_{p}(\omega)-\varepsilon_{m}}{\varepsilon_{p}(\omega)+2 \varepsilon_{m}}\right)+\frac{3\left(\varepsilon_{m} \sigma_{p}-\varepsilon_{p} \sigma_{m}\right)}{\tau_{M W}\left(\sigma_{p}+2 \sigma_{m}\right)^{2}\left(1+\omega^{2} \tau_{M W}^{2}\right)}=\Re_{\infty}+\left(\Re_{0}-\Re_{\infty}\right)\left(1+\omega^{2} \tau_{M W}^{2}\right)^{-1}
$$


where $\sigma$ denotes the electric conductivity [13]. At high frequency, $\omega>>\tau_{M W}^{-1}=\frac{\sigma_{p}+2 \sigma_{m}}{\varepsilon_{p}+2 \varepsilon_{m}}$ and $\mathfrak{R} \rightarrow \mathfrak{R}_{\infty}=\left(\frac{\varepsilon_{p}(\omega)-\varepsilon_{m}}{\varepsilon_{p}(\omega)+2 \varepsilon_{m}}\right)$. At low frequency, $\mathfrak{R} \rightarrow \mathfrak{R}_{0}=\left(\frac{\sigma_{p}(\omega)-\sigma_{m}}{\sigma_{p}(\omega)+2 \sigma_{m}}\right)$.

In the case of metallic particles, $\Re=1$ because the conduction band electrons continually redistribute themselves to reduce the internal electric field to zero. Then, taking a gap distance of $100 \mu \mathrm{m}$ in liquid nitrogen $\left(\Re e\left(\widetilde{\varepsilon}_{m}\right)=1.43\right)$, if $a=10 \mathrm{~nm}$, one finds at $10 \mathrm{kV}\left|\vec{F}_{d e p}\right| \approx 4 p N$, which is typically the order of magnitude found in other works (see for instance reference [14]) where aggregation occurs. This assumption also means that the dielectrophoretic force is not material-dependent because all metals behave alike.

On the other hand, Liao and co-workers [15] suggested a mechanism for the assembly of linear aggregates involving the formation of asymmetric charges on individual particles which assemble to become dimers. The integrative dipole moment of a dimer is greater than a single particle and therefore attracts another nanoparticle of cadmium more strongly, causing growth of the chain. Placed in an electric field, dipoles orientate along the field lines and bond by electrostatic interaction. This mechanism is possible with neutral particles that are mainly present in the space and time afterglow of the discharge.

\subsection{Proposed mechanism}

In every nano-object, the presence of elementary bricks made of nanoparticles of small size (5-10 nm for $\mathrm{Cd}$, and 30-50 $\mathrm{nm}$ for $\mathrm{Ag}$ ) is observed. Two unreported studies were carried out: one with $\mathrm{Cd}-\mathrm{Cd}$ electrodes and one with $\mathrm{Ag}-\mathrm{Ag}$ electrodes, from which we could draw the following conclusions. In the case of cadmium, particles are more elongated if the applied electric field is higher. It is not true for silver clusters, whose dendritic shape is very common. Cadmium particles appear after a period that depends on the applied voltage. At $10 \mathrm{kV}$, they are only visible after several hundreds of nanoseconds, but at $4 \mathrm{kV}$ they are visible from 100 ns on. On the contrary, whatever the applied voltages, silver clusters are synthesized only after several hundreds of nanoseconds. So, we readily admit that growth mechanisms are different for each element.

In both cases, elementary bricks are synthesized first, probably by condensation of the corresponding metallic vapor, since their mean size is set around specific values. However, these bricks get assembled differently. By optical emission spectroscopy, the intensity of emissive neutral species during the discharge is found to decay slowly, whereas ionic cadmium lines decay more rapidly and follow the applied current.

The influence of the electric field on the growth of cadmium objects suggests that elementary bricks are sensitive to this electric field, which is not the case of silver. The electric field can only act on the linear assembly once nanoparticles are formed, i.e. a short while after breakdown.

As explained previously, the frequency required to assemble metallic nanoparticles of a few tens of nanometers by dielectrophoresis must be in the $\mathrm{MHz}$ range. In the present situation, the ac current generated by the nanosecond pulse (see oscillations in Fig. 3a) is around 20 MHz. Nevertheless, if such a mechanism was effective, it would apply not only for cadmium but also for silver. Furthermore, the force would be higher for silver as the mean size of silver 
particles is larger than that of cadmium particles (see Eq. 1). Therefore, we propose to rule out this mechanism.

Concerning the possibility of assembling nanoparticles as dipoles requires to introduce a difference between cadmium and silver. We suggest that the initial asymmetry of charge distribution would be due to the non-spherical shape of the cadmium nanoparticles (Fig. 5c), contrary to silver particles. McCreery and Greenside [16] calculated a numerical approximation to the non-uniform surface charge density $\sigma$ for a charged conducting equipotential cubic surface in electrostatic equilibrium (Fig. 6a). Because of their crystallography, cadmium nanoparticles are prone to non-uniform charging and then, to linear assembly by dipole-dipole interaction.

This situation assumes that the assembly of two Cd nanoparticles is only possible if the area on one cube where the negative charge is mainly located faces the area of the other cube where the positive charge is mainly located. This requires a shift by a half of the cube edge length, as depicted in Fig. 6b. Such a shift is observed at high magnification (Fig. 6c).

Because the time required to set thermal processes is by far much longer than the discharge duration (hundreds of $\mu \mathrm{s}$ versus hundreds of ns), we also rule out any transport mechanism driven by heat transfer.
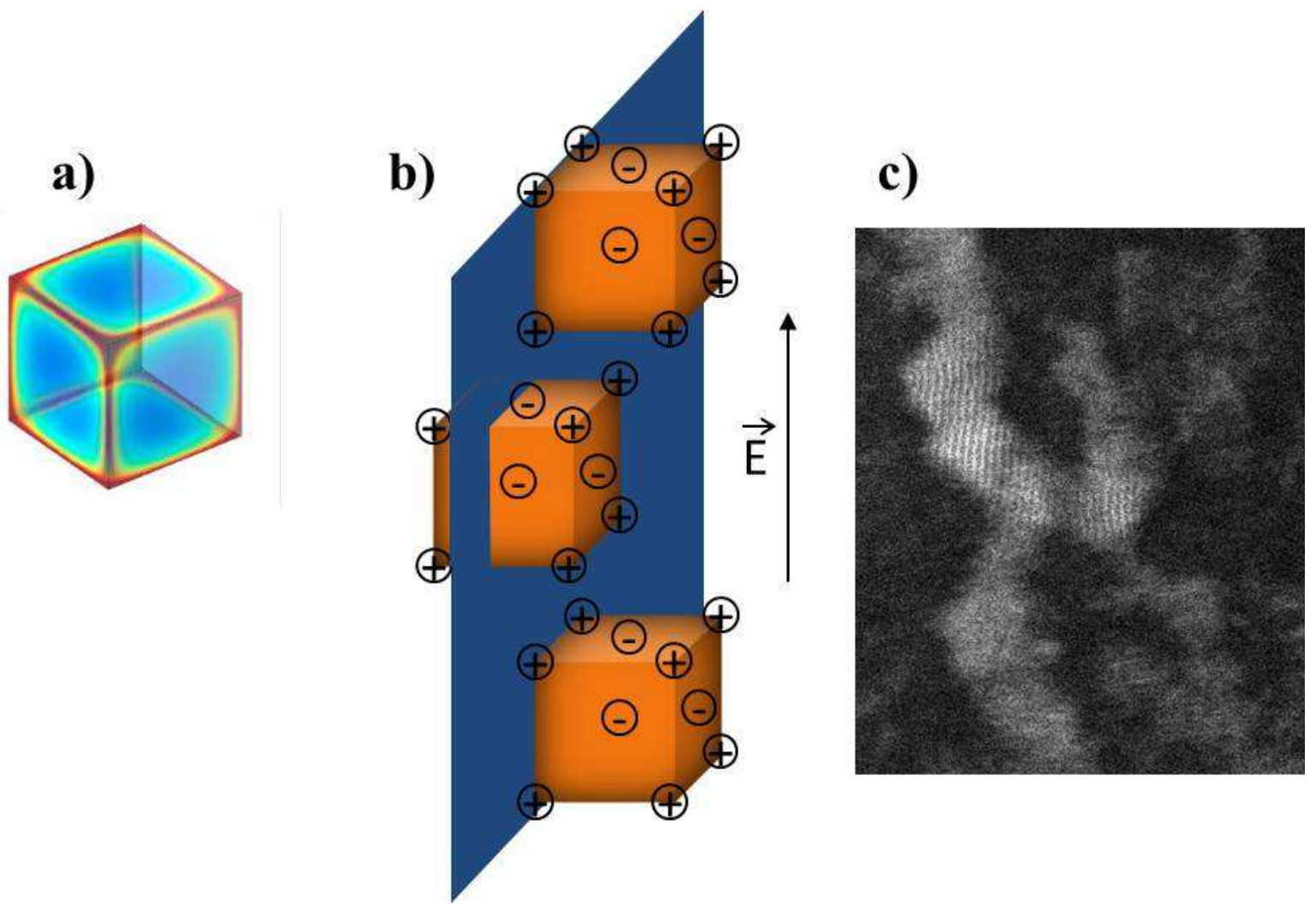

Fig. 6: a) Color density plot by McCreery and Greenside of the non-uniform surface charge density on the faces of a conducting equipotential cubic surface [16]. Reproduced with permission from Elsevier. b) Schematic illustration of the self-assembly process of metallic $\mathrm{Cd}$ nanoparticle chains based on the dipole assembly model by Liao et al. [15]. c) Magnification of a $\mathrm{Cd}$ wire showing the shift by a half edge length between primary cubic particles 
In our view, the formation of the elementary bricks happens in the discharge. There are two main stages:

- the synthesis of elementary bricks,

- their assembly to form wires or particles.

The role of the applied voltage is not only related to the polarization of the bricks but also to their density. When the applied voltage increases, the erosion of the electrodes is stronger (higher current flux), leading to more bricks. The overall kinetics of their formation is enhanced. This only applies if we assume that within the limited voltage range used for experiments (from 4 to $10 \mathrm{kV}$ ), the pressure distribution in space and time is not strongly affected.

On the other hand, their assembly starts when their concentration is sufficiently high. If the applied voltage is high, the moment happens earlier (or the distance to the plasma axis is shorter). The remaining electric field being higher, Cd-particles tend to be elongated, the transition from 2D (no-zero electric field) to 3D (zero electric field) being delayed. In Fig. 4, we do observe that elongated cadmium particles are produced at $500 \mathrm{~ns}$ at $10 \mathrm{kV}$ but they are still cubic at $4 \mathrm{kV}$.

Assembly of silver nanoparticles into 2-dimensional dendritic structures is assumed to be due either to surface diffusion when nanoparticles have fallen onto the substrate or to the existence of pressure gradients that enable diffusion only within iso-pressure layers, leading to 2D-objects. Synthesis from pure silver electrodes leads to stacked 2D-plates (see Supplemental material 11). Surface diffusion is assumed to be very limited because the substrate is at $77 \mathrm{~K}$. The other process, due to pressure gradients, is more likely. The presence of cadmium might be indirectly responsible for a change in the assembly process of silver particles (e.g. different discharge temperature or pressure), leading the formation of dendritic instead of dense structures.

The fact that nano-objects remain unalloyed, made of pure elements, suggests that vapors do not mix. The structure of the discharge is likely such that cadmium and silver vapors react close to the electrode where they are produced. Picosecond iCCD images, filtered in wavelength, are now scheduled to check this assumption.

\section{Conclusion}

In this work, we showed that the synthesis of nano-objects by discharges between one silver and one cadmium electrode cannot lead in our conditions to the formation of alloys. Elementary bricks have cubic-like shapes for cadmium and are spherical for silver. The emission of ionic and neutral lines of cadmium is useful to follow the dynamic of the discharge and to compare it to afterglow time. Both have similar lifetimes. For instance, for a pulse width of $100 \mathrm{~ns}$, the discharge and the afterglow last for about $250 \mathrm{~ns}$. We could not detect any ionic line of silver, probably because of excitation pathways leading to atomic levels are higher than the ion ground state. Modelling transition lines is under way but it is a complicated task. It will be useful to have access to plasma parameters.

The assembly of cubic-like Cd bricks into wires or elongated particles is attributed to a dipole-dipole interaction mechanism made possible thanks to the shape of the bricks that enables a non-uniform charge distribution. The possibility of dielectrophoresis is ruled out. Silver bricks form dendritic agglomerates.

\section{$7 \quad$ Acknowledgement}


This work benefited from the support of the project CEENEMA ANR-15-CE05-0005-01 of the French National Research Agency (ANR). 


\section{REFERENCES}

1 Toshima N, Yonezawa T. Bimetallic nanoparticles-novel materials for chemical and physical applications. New Journal of Chemistry, 1998, 22(11):1179-1201

2 Alayoglu S, Nilekar A U, Mavrikakis M, Eichhorn B. Ru-Pt core-shell nanoparticles for preferential oxidation of carbon monoxide in hydrogen. Nature Materials, 2008, 7:333338

3 Kim S M, Lee Y J, Kim J W, Lee S Y. Facile synthesis of Pt-Pd bimetallic nanoparticles by plasma discharge in liquid and their electrocatalytic activity toward methanol oxidation in alkaline media. Thin Solid Films, 2014, 572: 260-265

4 Pootawang P, Saito N, Takai O, Lee S Y. Synthesis and characteristics of Ag/Pt bimetallic nanocomposites by arc-discharge solution plasma processing. Nanotechnology, 2012, 23(39):395602

5 Chang H, Kao M J, Jwo C S, Kuo C G, Yeh Y H, Tzeng W C. J. Preparation of Co/Ag nanocompound fluid using ASNSS with aid of ultrasonic orthogonal vibration. Alloys and Compounds, 2010, 504S:S376-S379

6 Kabbara H, Ghanbaja J, Noël C, Belmonte T. Synthesis of copper and zinc nanostructures by discharges in liquid nitrogen. Materials Chemistry and Physics, 2018, 207:350-358

7 Dibitonto D D, Eubank P T, Patel M R, Barrufet M A. Theoretical models of the electrical discharge machining process. I. A simple cathode erosion model. Journal of Applied Physics, 1989, 66(9):4095-4103

8 Hamdan A, Noel C, Kosior F, Henrion G, Belmonte T. Impacts created on various materials by micro-discharges in heptane: Influence of the dissipated charge. Journal of Applied Physics, 2013, 113(4):043301.

9 Hamdan A, Noël C, Ghanbaja J, Belmonte T. Comparison of aluminium nanostructures created by discharges in various dielectric liquids. Plasma Chemistry and Plasma Processing, 2014, 34(5):1101-1114

10 Belmonte T, Noël C, Gries T, Martin J, Henrion G. Theoretical background of optical emission spectroscopy for analysis of atmospheric pressure plasmas, Plasma Sources Science and Technology, 2015, 24:064003

11 Hermanson K D, Lumsdon S O, Williams J P, Kaler E W, Velev O D. Dielectrophoretic assembly of electrically functional microwires from nanoparticle suspensions. Science, 2001, 294(5544):1082-1086.

12 Bernard L, Calame M, Van Der Molen S J, Liao J, Schönenberger C. Controlled formation of metallic nanowires via $\mathrm{Au}$ nanoparticle ac trapping. Nanotechnology, 2007, 18(23):235202.

13 Lumsdon S O, Scott D M. Assembly of colloidal particles into microwires using an alternating electric field. Langmuir, 2005, 21:4874-4880

14 Ramos A, Morgan H, Green N G, Castellanos A. Ac electrokinetics: a review of forces in microelectrode structures. Journal of Physics D: Applied Physics, 1998, 31:2338-2353 
15 Liao J, Zhang Y, Yu W, Xu L, Ge C, Liu J, Gu N. Linear aggregation of gold nanoparticles in ethanol. Colloids and Surfaces A: Physicochemical and Engineering Aspects, 2003, 223:177-183.

16 McCreery, K, Greenside H. The electric field of a uniformly charged non-conducting cubic surface. arXiv, 2016, 1607.07703. 